\title{
REFLEXIONES SOBRE LA INVESTIGACIÓN Y LA PRÁCTICA NARRATIVA EN TRABAJO SOCIAL
}

\author{
Reflections on research and narrative practice in Social Work
}

JOAQUÍN GUERRERO MUÑOZ ${ }^{1}$

\begin{abstract}
Resumen
El propósito de este artículo es realizar una reflexión teórica sobre las aportaciones de la orientación narrativa en la práctica y en la investigación social. El enfoque narrativo en las ciencias sociales ha recibido la influencia de la sociolingüística, de la antropología o la psicología, y de otras teorías como el interaccionismo simbólico o el constructivismo social. En este trabajo se analizan las limitaciones y las posibilidades de la investigación narrativa, en un contexto científico en el que la investigación cualitativa se ha consolidado, a pesar del positivismo imperante. La orientación narrativa ha contribuido además a desarrollar una visión crítica de la práctica del trabajo social, de los discursos profesionales dominantes y de la relación entre el usuario y el trabajador social. El enfoque narrativo, a través de la generación de nuevas historias que facilitan el cambio personal y social, nos aproxima a las experiencias personales considerando que los relatos son creados por los actores sociales, y que éstos son el resultado de un proceso intencional y reflexivo mediado por la cultura.
\end{abstract}

Palabras clave: Narrativa, investigación, trabajo social, teoría, práctica.

\begin{abstract}
The purpose of this article is to carry out a theoretical reflection on the contributions of narrative in practice and social research. The narrative approach in the social sciences has been influenced by sociolinguistics, anthropology and psychology, and other theories such as symbolic interactionism and social constructivism. In this paper the limitations and possibilities of narrative research are discussed in a scientific context in which qualitative research has been consolidated, despite the prevailing positivism. The narrative approach has also helped to develop a critical view of the social work practice, the dominant professional discourse, and on the user and social worker relationship. The narrative approach, through the generation of new stories that contribute to personal and social change, brings us closer to personal experiences considering these stories are created by social actors, and they are the result of an intentional and reflexive process mediated by culture.
\end{abstract}

Key words: Narrative, research, social work, theory, practice. 


\section{Introducción}

El impulso de la orientación narrativa en las ciencias sociales durante el último tercio del siglo Xx, se explica en gran medida debido a la fortaleza del constructivismo social y del interaccionismo simbólico en la sociología, la antropología cultural y la psicología social. Otras aproximaciones más contestatarias, revisionistas -incluso reformistas en algunos casos- del pensamiento social como el feminismo o el relativismo, favorecieron la narrativa explorando de forma crítica los límites de la investigación social así como la dimensión sociopolítica del propio discurso científico. De una parte, la narrativa provocó una reconsideración activa del rol del investigador, situándolo en un plano más creativo, como generador de nuevas piezas de significado, esto es, en tanto que autor de una escritura que incluye mínimamente una traducción de la experiencia humana a una forma textual (Clifford, 1995: 43), que lleva consigo el desvelamiento de significados ocultos y la construcción de nuevos a través del tamiz de la interpretación. De otra parte, el investigador quedaba interpelado en todo lo referido a su propio estatus o condición, reevaluando los discursos científicos tradicionales que hacían una lectura dogmática del proceso que conducía al conocimiento y de cómo éste debía articularse para alcanzar la verdad. En este aspecto concreto, por ejemplo, el postestructuralismo y el postmodernismo, especialmente los trabajos de los filósofos M. Foucault y J. Derrida, influyeron decisivamente en el enfoque narrativo. Sin ir más lejos en el ámbito de la terapia o de la clínica, al cuestionar abiertamente ciertas suposiciones dominantes subyacentes en la psicología, y poner de relieve el poder, la subjetividad del discurso, la ética, las cuestiones relativas al significado e incluso a la «cultura terapéutica» que están presentes en la relación entre el profesional y el usuario, y en la forma en que ambos perciben la realidad (Besley, 2001, 2002; Polkinghorne, 2004).

Una primera idea fundamental de la orientación narrativa que deseo destacar en esta introducción, es que la experiencia humana queda inscrita en los relatos que contamos, en los textos que producimos acerca de lo que hemos vivido, vivimos o está por vivir. Pensar la vida como concatenación de hechos o experiencias que son narradas, es pensarla como un relato biográfico, lo que significa en definitiva que somos a un tiempo, desde una perspectiva socio-literaria, autores o «novelistas» y «lectores» de nuestra propia historia (Contrera, 2006: 146). Las historias que crean las personas sobre sus vidas, determinan tanto la atribución de significado a sus vivencias como la selección de aquellos aspectos de la experiencia que van a poder expresarse en una infinidad de variados formatos. 
Una segunda idea clave que me interesa subrayar, desde el punto de vista de la práctica social, y especialmente de la relación que se construye entre el usuario y el profesional, es que los relatos biográficos son además constitutivos o moldeadores de las vidas de las personas (White y Epston, 1993: 55). En el relato personal podemos encontrar referencias a hechos, realidades o experiencias que nos proveen de un información muy relevante acerca de quién o quiénes somos o desearíamos ser. A través de lo narrado, del relato de lo acontecido y vivido, accedemos de forma privilegiada por un lado a la anatomía característica de la identidad personal, y por otro, a la experiencia ontológica del ser-en-el-mundo, una experiencia que se hace en el tiempo o como lo expresaba P. Ricouer: «...el tiempo se hace tiempo humano en la medida en que se articula en un modo narrativo, y la narración alcanza su plena significación cuando se convierte en una condición de la existencia temporal» (Ricouer, 2005: 113).

Por último, una tercera idea básica que conviene remarcar en esta introducción, es que en la construcción narrativa de lo vivido, del relato acerca de la experiencia personal como se acaba de enunciar, se incorporan además códigos, símbolos, valores, creencias y representaciones socioculturales de todo tipo. Las producciones narrativas de la persona son realidades discursivas, ancladas histórica y socioculturalmente. Las narrativas se configuran para una determinada audiencia en cierto momento histórico e incorporan referencias a los valores y discursos presentes en una cultura particular (Riessman, 2008: $3)$. Lo que se manifiesta a través del lenguaje, como relato o narración autobiográfica, aunque tan sólo sea de una forma limitada y parcial puesto que no todo lo vivido puede ser sustituido por la palabra y no todo lo experimentado es susceptible de ser narrado, posee un sentido en la cultura, es una forma de conocer el mundo, y a nosotros mismos, culturalmente mediada. La narración se sitúa en la cultura y la historia narrada dice algo del autor, pero también de la sociedad en la que vive el narrador de la historia (McAdams, 2006: 272).

La narrativa es una forma fundamental que posee el ser humano de dar sentido a la experiencia. Tanto en contar historias, como en interpretar las experiencias relatadas, la narrativa se encuentra entre un mundo interior de pensamientos y sentimientos, y un mundo externo de acciones observables, estados y hechos tangibles. La creación de una narrativa, así como la comprensión de la misma, es un proceso activo y constructivo que depende tanto de recursos personales como culturales. Las historias pueden ser un poderoso medio para el aprendizaje y la obtención de una comprensión de los demás, ya que ofrecen un contexto de significado sobre aquello que uno no ha experimentado personalmente (Mattingly y Garro, 2000: 1). En la narración, 
entendida de esta manera, existe por tanto un flujo necesario y constante entre un contexto personal (subjetivo) y otro cultural (intersubjetivo), rebosante de significados muchos de los cuales son compartidos. Ambos mundos están interconectados y entrelazados. Organizamos nuestra experiencia, y nuestra memoria principalmente, a través de la narrativa, es decir, de una forma convencional trasmitida culturalmente de relatar hechos verosímiles, de procurar una versión de la realidad aceptable, comprensible, asumible, más que porque pueda ser verificada empíricamente porque responde a una necesidad concreta y porque es retenida en esquemas cognitivos de significado (Bruner, 1990; 1991) que tienen su origen en la propia cultura y no únicamente en la psicología individual.

\section{Luces y sombras de la investigación narrativa en trabajo social}

En sus orígenes las investigaciones narrativas en el ámbito de la sociología y la antropología sociocultural abrieron un campo de estudio muy fructífero acerca, por ejemplo, de la experiencia de la enfermedad y la discapacidad que procuraron importantes contribuciones teóricas al trabajo social. El concepto de disrupción biográfica desarrollado por M. Bury (1982), el de loss of self de K. Charmaz (1983) o la distinción entre disease, illness, y sickeness de A. Kleinman (1988), son una muestra evidente de ello. La clarificación de las relaciones entre identidad personal y self, entre la experiencia subjetividad de la enfermedad y el autoconcepto, o los vínculos entre subjetividad, factores culturales y estructura social presentes en los relatos biográficos de la enfermedad fueron claves para la comprensión de esta realidad humana (Pierret, 2003).

Los métodos narrativos, en las ciencias sociales, y también en el trabajo social, se han asentado en el territorio de la investigación cualitativa de una forma clara e incontrovertible en las dos o tres últimas décadas. En la investigación social la narrativa se ha incorporado como miembro de pleno derecho, a pesar de haber sido fuertemente contestada por el positivismo. En la investigación en trabajo social no hay lugar a dudas del papel decisivo que enfoque narrativo posee tanto en la teoría como en la práctica profesional. En los últimos tiempos los manuales, ensayos y textos especializados recogen en sus contenidos revisiones teóricas, aplicaciones y nuevos escenarios en los que se vislumbra un fructífero recorrido para esta orientación (Elliott, 2005; Fortune, Reid y Miller, 2013; Riessman, 2008; Shaw y Gould, 2001; Wells, 2011). En un trabajo reciente sobre esta temática, C. Baldwin, destaca varios campos en los que la narrativa, tanto desde el punto de vista de la práctica profesional, como de la investigación y la construcción teórica, está teniendo 
importantes y significativos resultados. La investigación narrativa en trabajo social, muchas veces en relación directa con determinadas experiencias que emanan de la práctica, ha dado lugar a debates muy interesantes acerca del estatuto de algunas creencias y prácticas profesionales asumidas de forma acrítica por los propios trabajadores sociales. Algunos de los ámbitos en los que se emplea la orientación narrativa son, por ejemplo, los derechos humanos y la justicia social, la ética del trabajo social, la política social y los discursos y prácticas en la protección infantil, la salud mental o la discapacidad (Baldwin, 2013), entre otros. La perspectiva narrativa ha demostrado su capacidad de suscitar nuevos interrogantes y de plantear reflexiones metodológicas y epistemológicas de gran calado a partir de la consideración de experiencias vitales radicales que provocan aislamiento social y un considerable deterioro de la identidad personal (Baldwin, 2006; Bouchard-Ryan, Bannister y Anas, 2009).

A pesar de lo dicho anteriormente, y de que el enfoque narrativo ha experimentado en los últimos años un gran empuje, la investigación en trabajo social discurre hacia otros derroteros y apenas deja un hueco aceptable para propuestas metodológicas más «arriesgadas» que se ubican en ese territorio incierto de lo cualitativo. Entre los profesionales del trabajo social existe una necesidad imperiosa de legitimar su actividad, dotándola de fortaleza «científica», y por tanto asemejándola al modelo experimental de las «ciencias duras» y a los estudios basados en la evidencia, como por otra parte vienen haciendo la psicología y la sociología desde hace bastante tiempo. Por ello existe una predisposición a rehuir los métodos y las orientaciones, que como la narrativa, tienen un encaje más difícil en el canónico método científico. Existen varias razones que pueden ayudarnos a comprender una situación paradójica que se vive en la actualidad. Por una parte la necesidad sentida por los trabajadores sociales de aproximarse a ciertas realidades con una «mirada» diferente, que ahonde en dimensiones de la experiencia humana que sólo afloran en la práctica y que son opacas desde una visión exclusivamente positivista, no naturalista, y por otra parte, el entorpecimiento constante -cuando no la censura o la propia autocensura- para que los estudios cualitativos de corte narrativo se muestren en las revistas especializadas de mayor impacto a un mismo nivel que los cuantitativos.

Las limitaciones metodológicas de la investigación narrativa que con frecuencia se esgrimen, falta de representatividad y de objetividad, ausencia de sistematización en los datos, imposibilidad para su replicación, dificultades para establecer criterios de fiabilidad y validez, etc., no han minado ni su atractivo, ni su valor y utilidad, tanto en la teoría como en la práctica del trabajo social. Una poderosa razón que sostiene el «feeling narrativo» en el 
trabajo social, es que esta disciplina se alimenta de los relatos y de las vivencias personales, son su materia prima, y la conversación, el intercambio comunicativo cercano, es el medio genuino en el que tiene lugar el encuentro con lo humano. Parafraseando una conocida expresión de N. Denzin, «sólo podemos disponer de las vidas mediante las palabras», diría que sólo podemos conocer al otro, y su mundo vital, a través de lo que nos cuenta de sí mismo, como un relato privilegiado de la experiencia humana.

Otro de los aspectos que se ha sugerido en algunos trabajos, sobre las limitaciones de la investigación narrativa se relaciona con la propia estructura organizativa de las universidades, ampliamente segmentadas y parcializadas en departamentos. En las instituciones universitarias los enfoques transdisciplinares, como el narrativo, encuentran trabas para sortear los límites visibles e invisibles de las fronteras disciplinares (Riessman y Quinney, 2005: 406). La investigación colaborativa, imprescindible si adoptamos un enfoque narrativo, es más un ideal que una realidad. Existen resistencias y dificultades de todo tipo que llegan a sorprender en un momento en el que precisamente la investigación multimétodo parece haberse popularizado en el mundo académico. En ocasiones la formación de postgrado resulta muy rígida y no permite habilitar los espacios o las pacerlas necesarias para introducir contenidos que a priori se distancian por su naturaleza de una formación más estandarizada o reglada comúnmente admitida por los profesionales y los expertos. Por último, las propias revistas científicas, sus editores, consejos científicos y revisores principalmente, asumen una consideración previa, cuando no un prejuicio manifiesto, acerca de lo que es o no científico, de lo que debería ser la ciencia, que choca frontalmente con la estrategia narrativa (Riessman y Quinney, 2005: 406).

Un ejemplo claro de esta limitación en la producción científica de corte narrativo en trabajo social es que en revistas como Qualitative Social Work e International Journal of Qualitative Methods, durante el periodo comprendido entre 2010 y 2014, de un total de 173 artículos publicados, un 19,6\% de los mismos empleaban la metodología narrativa, o se ocupaban de tratar cuestiones teóricas, epistemológicas, metodológicas y éticas relativas a la investigación narrativa en trabajo social. Es un porcentaje nada desdeñable. Sin embargo si lo comparamos con otras revistas de gran impacto, pero más generalistas, como Social Service Review de la Universidad de Chicago o Journal of Social Policy de la Universidad de Cambridge, para el mismo periodo de tiempo, hallamos que de un total de 255 artículos publicados, tan sólo dos de ellos empleaban la narrativa desde una perspectiva multimétodo. Esto supone en realidad menos del $1 \%$ de los artículos publicados, en concreto, un $0.7 \%$, 
lo cual parece dibujarnos un escenario bastante restrictivo para la investigación cualitativa en general, y de forma más explícita o singularizada para el enfoque narrativo en la investigación en trabajo social. Estos datos irían en la dirección de apoyar la tesis de Riessman y Quinney, anteriormente mencionada, sobre la baja o nula permeabilidad de algunas revistas científicas para hacer visibles propuestas de corte más cualitativo como el enfoque narrativo.

\section{Consideraciones sobre la práctica narrativa en trabajo social}

El uso de la narrativa, de lo que nos cuenta el usuario en una entrevista o conversación, su propia historia relatada, es un medio natural a través del cual el/la trabajador/a social (en adelante trabajador social) puede considerar la situación singular en la que se encuentra la persona con todas sus particularidades y matices. Las historias son la forma más auténtica de describir las experiencias vitales y sus significados, el andamiaje sobre el que se impone un cierto orden en el «caos» de la experiencia (Ollikainen, 2005:322). La narración del usuario, es un producto biográfico denso que cambia con el tiempo, se transforma, porque no es estático sino dinámico, está influido por la propia experiencia y los acontecimientos así como por las interpretaciones que la persona realiza sobre sí mismo, su situación presente, pasada y futura y por la relación que entabla con el propio profesional. La orientación narrativa es del todo un método de trabajo centrado en la persona. El trabajador social accede a través del relato al stock narrativo, es decir, a los significados culturales y personales profundos que emplea cada narrador (cada persona), como una señal inequívoca de su propia identidad (Ollikainen, 2005:326).

No existe una definición clara de qué se entiende por narrativa, ya que entre otras razones el enfoque narrativo es plural en sus perspectivas y en los principios teóricos que lo avalan. Riessman había hablado de la tiranía de la narrativa refiriéndose a la profusión de este término que en el lenguaje científico, hasta el punto que parecía significar cualquier cosa y todo a la vez (Riessman, 1997). En historia social y en antropología la narrativa se vincula con las historias de vida, mientras que en la sociolingüística por ejemplo el concepto de narrativa se refiere a historias, según el trabajo de N. Labov, que se organizan alrededor de un personaje, un escenario y una trama. En la psicología y la sociología se define la narrativa como los amplios segmentos de la charla que se produce en las entrevistas y que incluye la interacción entre el narrador y el entrevistador (Larsson y Sjöblom, 2010: 274). Por encima de estos matices, los investigadores reconocen ciertos elementos comunes. La narración se caracteriza por poseer un orden y una secuencia clara de acontecimientos y hechos; una acción se ve como consecuencia de la siguiente y estas 
acciones se relacionan con ciertas ideas o argumentos, por tanto, podríamos decir que la narración es contingente. Los narradores crean argumentos de una experiencia desordenada, confiriéndole realidad y coherencia. En relación con esto, los narradores estructuran sus relatos temporal y espacialmente y en ellos la acción humana y la imaginación se expresan vívidamente (Riessman, 2013: 172).

La utilidad de la narrativa en el trabajo social, desde la dimensión teóricopráctica, se comprende se consideramos los siguientes aspectos:

a. Es un medio para la exploración y el análisis de la identidad personal, que podemos emplear con fines diagnósticos, terapéuticos o de intervención. En el trabajo social los relatos personales son un género particularmente significativo que estimula el conocimiento de la identidad personal. Los métodos narrativos favorecen el estudio sistemático de la experiencia humana, y por medio de ésta conocemos cómo son las personas, por qué se comportan o actúan de un modo determinado, cuáles son sus intenciones, motivaciones, aspiraciones, frustraciones, etc. La narración personal nos orienta sobre el humano vivir, y la práctica social se humaniza en la escucha y en la comprensión del discurso ajeno. La atención a la narrativa personal en la investigación cualitativa, a través por ejemplo de las entrevistas, genera espacios discursivos únicos, en los sujetos de investigación se muestran como edificadores de su propia experiencia y como agentes que actúan en los escenarios de la vida de una gran complejidad moral. (Riessman, 2013: 185). La exploración de la identidad personal permite realizar un diagnóstico de la situación teniendo en cuenta la imagen que las personas tienen de sí mismas y de la realidad, desde su propio universo valorativo y cultural.

b. Nos obliga a repensar la relación entre el usuario y el profesional donde la escucha, el compromiso y la interpretación juegan un rol decisivo en la humanización del proceso de ayuda. Para el trabajador social la producción narrativa es fundamental. Favorece en cualquier caso una visión de la persona desde el interior. Contribuye además a realizar propuestas, tomar decisiones y llevar a cabo acciones que se asientan en la experiencia vivida de modo concreto. En cierto sentido se ha indicado que la narrativa evita la desaparición de la persona (Booth, 1998: 256), en la intervención. En ocasiones esta desaparición de la persona tiene lugar cuando existe una teorización y abstracción muy elevadas, o bien, cuando se emplean todo tipo de tecnicismos para 
encubrir o enmascarar una realidad, la propia experiencia, que supera una definición objetiva preestablecida o definida por el experto. Se pone el acento en lo que verdaderamente es importante para la persona, y no tanto en aquello que el experto o el profesional considera decisivo, relevante o valioso.

c. Por último, la narrativa describe sus propias coordenadas para la intervención dirigida al cambio a partir de la re-escritura del relato personal. El relato es transformador, no únicamente un medio para tomar conciencia de algo, reflexionar acerca de una cuestión determinada o traer recuerdos a la memoria, sino que permite a la persona distanciarse del problema, objetivarlo, o externalizarlo como sugieren M. White y D. Epston (1993), para asumir el control sobre él y entonces estar en disposición de descubrir «acontecimientos extraordinarios»-como estos autores los denominan-, nuevos significados, formular otras posibilidades o escoger entre diferentes alternativas rompiendo así el círculo vicioso entre la persona y la narración centrada en sucesos y experiencias vitales negativos. Cuando un usuario acude a un servicio la historia que nos relata está saturada por el problema, esto es, existe un argumento reiterativo y dominante (Morgan, 2002) en torno al cual giran todos los hechos y acontecimientos biográficos. Este relato está cosificado, impide ver alternativas y habitualmente se edifica sobre experiencias previas de fracaso. Estas experiencias refuerzan poderosamente una expectativa negativa acerca de lo que el futuro puede deparar, convirtiéndose el fracaso en la profecía autocumplida. La persona está «dominada» por su propio relato. El propósito de la intervención es justamente devolverle su capacidad de agencia personal, invitándole a tomar distancia del problema y a generar nuevos relatos, cuestionando ideas preestablecidas que hasta el momento no se habían puesto en duda, y que por tanto tenía un carácter dogmático. Un ejemplo de aplicación práctica de este enfoque lo tenemos en la propuesta de K. D. Roscoe, A. M. Carson y L. Madoc-Jones (2011). El modelo de intervención que sugieren estos autores está basado en tres etapas: 1) el compromiso con el usuario del servicio, 2) la exploración y la deconstrucción de las historias saturadas por el problema y 3) el trabajo con los usuarios para la re-escritura de las historias. El compromiso es la clave del éxito de la intervención y las habilidades para el compromiso son un componente vital del trabajo social: aceptación incondicional del relato, confianza mutua y empatía básicamente. La relación no está jerarquizada, si se enmarca en la lógica del poder 
del experto que impone su criterio. En las conversaciones entre el trabajador social y el usuario, ambos pueden participar compartiendo historias sobre el supuesto inicial de que poseen el mismo valor. El respeto mutuo es una parte constitutiva de este tipo de compromiso. Permite además ver cuál es la situación real del otro. La exploración de las historias o de los relatos del usuario, lleva consigo un proceso de cuestionamiento y deconstrucción de las ideas previamente asumidas. Este es el segundo paso de la intervención. Algunos de los participantes en estos encuentros pueden tener una visión particular de la realidad que ahora necesitan cuestionar, y para ello se estimulan las preguntas que predisponen a una reflexión compartida dirigida a favorecer un cambio positivo: ¿cómo está afectando el problema a la imagen que tienes de ti mismo?, ¿cómo han variado tus relaciones sociales?, ¿cómo influye la situación en tu vida cotidiana?, etc. Por último la re-escritura de la historias es una invitación a crear otras narrativas que incluyen tanto al trabajador social como al usuario en el desarrollo de una comprensión mutua, que da lugar a un escenario relacional en el que el profesional estimula la búsqueda activa de acontecimientos que contradicen el argumento dominante del relato.

\section{Conclusiones}

Pese al interés que el enfoque narrativo ha despertado tanto en la práctica como en la investigación en trabajo social, sin embargo, podemos concluir que su desarrollo y consolidación están determinados por diversos factores. A continuación se enumeran algunos aspectos críticos con relación a la investigación narrativa:

- Cuando nos referimos a la investigación narrativa, ésta queda supeditada la mayoría de las veces a las técnicas e instrumentos de investigación, corriendo el riego evidente de un grave reduccionismo metodológico y epistemológico. Una investigación narrativa no lo es únicamente porque emplea instrumentos de recogida de datos como las historias de vida o las entrevistas en profundidad. La narrativa implica además una «mirada», un enfoque teórico y una perspectiva de análisis diferentes. El enfoque narrativo, al igual que la etnografía por ejemplo, es fenomenológico y hermenéutico, como ya se ha visto, en tanto que asume por un lado que las acciones humanas se basan y están inducidas por significados sociales: intenciones, motivos, actitudes y creencias (Hammersley y Atkinson, 2001: 21), y por otro, que 
el relato mismo es el contexto en el que el comportamiento humano se hace inteligible, por tanto susceptible de ser desvelado a través de la interpretación.

- La orientación narrativa no es un modelo teórico en el sentido en el que habitualmente empleamos este término en trabajo social, aunque evidentemente se nutre de la teoría y sus presupuestos reposan en ella. Las corrientes teóricas que han influido en la narrativa son múltiples, lo cual ha conducido al surgimiento de distintas tradiciones como la sociolingüistica, el estructural-funcionalismo, el cognitivismo o el constructivismo, que se aplican en uno y otro caso dificultando la comparación de los resultados procedentes de las distintas investigaciones. Tampoco la definición de un lenguaje teórico común, más o menos unificado y de amplio consenso entre los expertos, se ha podido gestar. Se añade además que se emplean diversos procedimientos y marcos teóricos explicativos para el análisis de los datos. Este eclecticismo produce una disfunción metodológica importante, ya que una misma evidencia narrativa puede derivar hacia conclusiones diametralmente opuestas, en base al enfoque o la estrategia teórica empleada para su «disección».

- Cabe indicar también que la investigación narrativa ha sucumbido al efecto de las técnicas testimoniales, favoreciendo la psicologización de los resultados, en detrimento de la sistematización, tanto en la recolección de los datos como en el análisis de los mismos. Se ha impuesto el valor de la autoría narrativa, el de la persona que cuenta su historia o relato, incluso por encima de las estrategias que permiten alcanzar una mayor fiabilidad y validez a la investigación, todo ello bajo el lema de que el testimonio narrativo por sí mismo es representativo y valioso. Muchos trabajos han caído en un realismo sentimental de caso único, concediendo un papel nuclear a los sentimientos y experiencias personales que son recogidas en forma de una escritura, ya no científica, sino puramente biográfica o auto-biográfica, que difícilmente puede responder a unos mínimos parámetros de contrastación o generalización (Atkinson, 2013: 19). Este es un gran riesgo para la investigación narrativa, que puede tornarse en un ejercicio literario sin pretensiones científicas.

- Otro aspecto a tener en consideración es que, por un lado, la formación académica en lo que se refiere a los procedimientos narrativos aplicados a la investigación es muy débil, cuando no inexistente en prácticamente todas las etapas formativas en la enseñanza 
universitaria, y por otro lado, el habitual desconocimiento que los profesionales y la propia comunidad científica en el ámbito del trabajo social poseen de los mismos.

- No podemos obviar tampoco la existencia de un pudor casi visceral y atávico a distanciarse de patrones estandarizados por la investigación «convencional», difícilmente explicable si no atendemos a la presión que se ejerce desde el ámbito académico -y desde las propias instituciones y organismos encargados de la difusión científica- por implementar y auspiciar trabajos que representarían el ideal de un racionalismo empirista propio de las ciencias «duras». Esta circunstancia hace que los investigadores tiendan a proponer diseños con mayores expectativas de éxito, desde el punto de vista de la estrategia en la captación de recursos económicos, que además posibiliten finalmente la viabilidad del estudio y su «rentabilidad» en términos de impacto científico y difusión.

Si atendemos ahora a la práctica profesional enmarcada en un enfoque narrativo, sería necesario destacar también, a modo de conclusión, algunas de sus limitaciones y aportaciones más sobresalientes. Comenzaré destacando positivamente algunos aspectos, dejando para el final algunas cuestiones relacionadas con la práctica que están por definir adecuadamente y desarrollar en profundidad:

- En primer lugar, los métodos narrativos son multidimensionales y tratan de capturar una versión más compleja de la existencia humana (Larsson y Sjöblom, 2010: 279), lo que encaja muy bien con las pretensiones teóricas y prácticas del trabajo social. En el ámbito del trabajo social con grupos, por ejemplo, y en aquellos casos en los que nos encontramos con una orientación claramente clínica o terapéutica, puede que más propia del escenario en el que habitualmente se mueven los profesionales de la salud, la utilización de la narrativa del usuario constituye por así decirlo el punto de partida en el que se inicia el proceso curativo y/o rehabilitador, esto es, el punto de inflexión en el que acontece el cambio personal y social (Guerrero, 2013). La orientación narrativa implica un verdadero «giro», que P. Abels y $S$. L. Abels (2002) ya habían considerado en la intervención con grupos. Esta nueva perspectiva viene a sentar las bases de la «mirada narrativa» en el trabajo social. Insisto en el término «mirada» porque en realidad se trata precisamente de eso, de la perspectiva que asumimos ante la realidad y la problemática personal. El enfoque narrativo 
ayuda a combatir una visión de la realidad excesivamente determinada, la que impone la racionalidad científica, para otorgar vía libre a las ambigüedades, las paradojas, las contradicciones que caracterizan la vida y la experiencia cotidiana de las personas (Booth, 1998: 258).

- Los relatos no sólo nos dan información sobre nosotros mismos, los demás y el mundo, sino que la narrativa, como perspectiva que guía la intervención y proceso, también da forma a lo que experimentamos y nos permite impregnar el mundo con significado (Baldwin, 2013: 133). La narrativa en la práctica nos proporciona información acerca de la persona, de sus relaciones sociales y de su entorno cultural. Las personas cuentan historias a otras personas, de acuerdo con ciertas expectativas sociales y normas, y cualquier expresión narrativa no puede entenderse fuera del contexto del narrador y del oyente, con relación al cual la historia se ha diseñado al objeto de trasladar una información que producirá un determinado efecto (McAdams, 2008: 245). Las narraciones reflejan también la cultura en la que se han creado y transmitido a los demás. El relato ha de considerarse como algo más que una simple biografía, apunta en todo momento a la situación o al contexto histórico y cultural en el que se articula, lo cual implica necesariamente que el trabajador social lleve a cabo a través del enfoque narrativo una práctica culturalmente competente.

- La relevancia de la narrativa para la práctica en el trabajo social se manifiesta por su papel central en la conformación de la identidad personal (Wilks, 2005: 1252), y por tratarse de un medio natural de acceso al otro, a los significados que nos hablan acerca de cómo percibe la realidad y le confiere un determinado sentido. Observar la identidad personal desde la mirada narrativa hace posible que la entendamos como algo dinámico, no como un constructo fijo o estático. El relato personal reúne a las diversas fuentes de la identidad y les da coherencia y sentido a través de la historia que contamos (Wilks, 2005: 1254). De ahí que la narración sea decisiva en el proceso de escucha, cuando atendemos la demanda del usuario y comenzamos a construir los cimientos de una relación de ayuda basada en el compromiso. Entiendo al otro, por lo que el otro es, por medio del relato, a partir de aquello que me cuenta sobre su vida, acerca de su experiencia singular, sin pretender imponer una visión concreta de la realidad. El relato es, igualmente, la resultante de un proceso intencional y reflexivo (Roscoe, Carson y Madoc-Jones, 2011: 50), del que nace creativamente que representa amplias parcelas de la identidad personal. 
- El enfoque narrativo nos previene de los apriorismos que abundan en el discurso profesional, y del abusivo e indiscriminado uso que en ocasiones se realiza de los conceptos teóricos y técnicos del trabajo social a la hora de identificar, definir, diagnosticar, explicar y comprender una determinada realidad humana. Los trabajadores sociales no son ajenos a los sesgos o a los prejuicios que a menudo interfieren en la relación de ayuda. Cómo escuchamos los problemas de las personas que acuden a un servicio y qué valor le conferimos a lo que nos cuentan condiciona enormemente la forma en la que los abordamos. La narrativa nos libera en cierto modo de una actitud acrítica y condescendiente, y nos facilita poner en práctica métodos innovadores y creativos que rompen con prácticas profesionales que se distancian del discurso personal, parapetándose en una visión estandarizada, e incluso opresiva, de la relación usuario-profesional (Roscoe, 2009: 11). Al aceptar incondicionalmente el relato que nos proporcionan las personas, les reconocemos como autores de sus propias vidas y nos ponemos en disposición de atender a lo que realmente nos cuentan, y no tanto a lo que esperamos o deseamos oír.

- El propósito de la práctica profesional desde el trabajo social sería el de facilitar que las personas pudieran generar historias alternativas, optimizando todos sus recursos. Para lo cual se requiere cultivar con el usuario la auto-reflexión, identificando en el relato hechos que contradicen la historia principal, o el argumento dominante sobre el que se ha construido (Freedman y Combs, 1996), y sobre ellos generar nuevos relatos que operarán a partir de las excepciones a la regla, esto es, a la historia saturada por el problema. Este tipo de innovación narrativa, impulsa el cambio en sí mismo y el posicionamiento de la persona como autora de un nuevo relato, con autonomía y libertad para dotar de nuevos sentidos a sus experiencias (Gonçalves, Matos y Santos, 2009).

- La práctica narrativa, a pesar de ser una estrategia que desde el punto de vista profesional es acogida de buen grado, en cambio adolece de herramientas consistentes de diagnóstico evaluación de los objetivos y resultados, y tampoco existen evidencias claras de que la sustitución de ciertos relatos dominantes por otros alternativos, produzca un cambio sostenible en el tiempo, y lo que es más difícil aún, un cambio que suponga una auténtica transformación en el entorno social y por extensión en los discursos más generales de los que se nutren por ejemplo las propias instituciones y organizaciones. 
- Un último aspecto a considerar es la falta de evidencias que nos proporcionen una estimación objetiva de la utilidad de este enfoque, frente a otras estrategias centradas en modelos ya clásicos del trabajo social como por ejemplo el cognitivo-conductual o el ecológico-sistémico. No contamos con el apoyo de estudios que se hayan ocupado de este asunto en profundidad, y que de forma comparativa nos muestren las fortalezas y debilidades de la orientación narrativa en relación con otros planteamientos.

Finalmente, la indagación pormenorizada en los aspectos que afectan a la metodología narrativa abre nuevos horizontes para la investigación y la práctica en trabajo social. Sin embargo, por el momento queda mucho camino por recorrer hasta lograr, podríamos decir, una competencia narrativa entre los profesionales, que hasta el día de hoy no deja de estar poco, o nada desarrollada en nuestros planes formativos tanto de grado como de postgrado.

\section{Bibliografía}

ABELS, P. y ABELS, S. L. (2002). «Narrative social work with groups: just in time». En S. Henry, J. East, y J. Schmitz, J. (Coords.). Social work with groups: mining the gold. (pp. 57-73). New York: Haworth Press.

AtKinson, P. (2013). Ethographic writing, the Avant-Garde and failure of nerve. International Review of Qualitative Research, 6 (1), 19-35.

BALDWIN, C. (2006). «The narrative dispossession of people living with dementia: thinking about theory and the method of narrative». En Milnes, K., Horrocks, C., Kelly, N., Roberts, B. y Robinson, A. (Eds.). Narrative, Memory and Knowledge: Representations, Aesthetics, Contexts. (pp. 101-109). Huddersfield: University of Huddersfield Press.

BALDwIN, C. (2013). Narrative social work. Bristol: The Policy Press.

BESLEY, T. (2001). Foucauldian influences in Narrative Therapy: an approach for schools. Journal of Educational Enquiry, 2 (2), 72-93.

BESLEY, T. (2002). Foucault and the turn to narrative therapy. British Journal of Guidance \& Counselling, 30 (2), 125-143.

Воотн, Т. (1998). «El sonido de las voces acalladas: cuestiones acerca del uso de los métodos narrativos con personas con dificultades de aprendizaje». En L. Barton (Comp.). Discapacidad y Sociedad. (pp. 253-271). Madrid: Morata.

BOUCHARD-RYAN, E., BANNISTER, K. A. y ANAS, A. P. (2009). The dementia narrative: writing to reclaim social identity. Journal of Aging Studies, 23, 145-157.

BRUNER, J. (1990). Acts of meaning: four lectures on mind and culture. Cambridge, MA: Harvard University Press. 
BRUNER, J. (1991). The narrative construction of reality. Critical Inquiry, 18 (1), $1-21$.

Bury, M. (1982). Chronic illness as biographical disruption. Sociology of Health and Illness, 4 (2), 167-182.

CHARMAZ, K. (1983). Loss of self: a fundamental form of suffering in the chronically ill. Sociology of Health and Illness, 5 (2), 168-195.

Clifford, J. (1995). Dilemas de la cultura. Antropología, literatura y arte en la perspectiva posmoderna. Barcelona: Gedisa.

Contrera, V. (2006). La narrativa en trabajo social: entrevista familiar como espacio de re-construcción de relatos. Revista Tendencias y Retos, 11, 143-151.

ELLIOTT, J. (2005). Using narrative in social research. Qualitative and quantitative approaches. London: Sage.

FREEDMAN, J. Y COMBS, G. (1996). Narrative therapy: the social construction of preferred realities. New York: Norton.

Fortune, A. E., ReID, W. J., y Miller, R. (2013). Qualitative research in social work. New York: Columbia University Press.

Gonçalves, M., Matos, M., y Santos, A. (2009). Narrative therapy and the nature of «innovative moments» in the construction of change. Journal of Constructivist Psychology, 22 (1), 1-23.

Guerrero, J. (2013). Recursos narrativos para el trabajo social con grupos. Humanismo y Trabajo Social, 12, 95-108.

Hammersley, M. y AtKinson, P. (2001). Etnografía. Métodos de investigación. Barcelona: Paidós.

Kleinman, A. (1988). The illness narratives. Suffering, healing and the human condition. New York: Basic Books.

LARSSON, S. y SJÖBlOM, Y. (2010). Perspectives on narrative methods in social work research. International Journal of Social Welfare, 19, 272-280.

Mattingly, CH. y Garro, L. C. (2000). «Narrative as construct and construction». En Ch. Mattingly y L. C. Garro, (Eds.). Narrative and the cultural construction of illness and healing. (pp. 1-40). Berkely, LA: University of California Press.

MCADAMS D. P. (2006). The redemptive self: stories americans live by. Oxford: Oxford University Press.

McADAms, D. P. (2008). «Personal Narratives and the Life Story». En O. P. John, R. W. Robins, y L. A. Pervin, (Eds.). Handbook of Personality: theory and research. (3. ${ }^{a}$ ed., pp. 242-262). New York: Guilford Press.

Morgan, A. (2002). What is narrative therapy?. Adelaide: Dulwich Centre Publications.

OllikAinem, P. (2005). «Use of narratives in psychosocial work with cancer patients». En T. Heinonen y A. Metteri (Eds.). Social work in health and mental 
health: issues, developments and actions. (pp. 321-337). Toronto: Canadian Scholars Press.

PIERRET, J. (2003). The illness experience: state of knowledge and perspectives for research. Sociology of Health and Illness, 25, 4-22.

POLKINGHORNE, D. E. (2002). «Narrative therapy and postmodernism». En L.

E. Angus y J. McLeod (Eds.). The handbook of narrative and psychotherapy. Practice, theory and research. (pp. 53-67). London: Sage.

Ricouer, P. (2005). Tiempo y Narración I. Configuración del tiempo en el relato histórico (5. ${ }^{a}$ ed.). Buenos Aires: Siglo XXI.

RIESSMAN, C. K. (1997). A short story about long stories. Journal of Narrative and Life History, 7 (1-4), 155-159.

RIESSMAN, C. K. y QUINNEY, L. (2005). Narrative in Social Work. A critical review. Qualitative Social Work, 4 (4), 391-412.

RIESSMAN, C. K. (2008). Narrative methods for Human Sciences. New York: Sage. RiEsSMAN, C. K. (2013). «Analysis of personal narratives». En A. Fortune, W. J. Reid, y R. Miller (Eds.).Qualitative Research in Social Work. (pp. 168-191). New York: Columbia University Press.

RosCoE, K. D. (2009). Critical social work practice a narrative approach. International Journal of Narrative Practice, 1 (1), 9-18.

RosCoE, K. D., CARSON, A. M., y MADOC-JONES, L. (2011). Narrative social work: conversations between theory and practice. Journal of Social Work Practice, 25, 47-61.

SHAW, I. y GOULD, N. (2001). Qualitative research in social work. London: Sage.

WeLLS, K. (2011). Narrative Inquiry. Oxford: Oxford University Press.

White, M. y Epston, D. (1993). Medios narrativos para fines terapéuticos. Barcelona: Paidós.

WiLKS, T. (2005). Social work and narrative ethics. British Journal of Social Work, $35,1249-1264$. 